\title{
A successful full term delivery in a case of robotic assisted insertion of transabdominal cerclage in an obese woman with multiple uterine fibroids
}

\author{
Y. H. Tan*, S. Durai, K. Devendra, N. Ravichandran
}

Department of Obstetrics and Gynaecology, Singapore General Hospital, Singapore

Received: 17 October 2020

Revised: 15 December 2020

Accepted: 03 February 2021

*Correspondence:

Dr. Y. H. Tan,

E-mail: yinghao.tan@mohh.com.sg

Copyright: (C) the author(s), publisher and licensee Medip Academy. This is an open-access article distributed under the terms of the Creative Commons Attribution Non-Commercial License, which permits unrestricted non-commercial use, distribution, and reproduction in any medium, provided the original work is properly cited.

\begin{abstract}
Cervical incompetence is not an uncommon presentation in an obstetric emergency unit. Some of these patients will be managed with a cervical cerclage. While the management of patients presenting with first time cervical incompetence is relatively established, the management of patient with repeated cervical incompetence might require an abdominal cerclage. Abdominal cerclages can be inserted traditionally via laparotomy or via a minimally invasive approach (MIS). We present a case of an obese patient presenting with 3 previous second trimester miscarriages despite 2 cervical cerclage complicated by multiple uterine fibroids who underwent a robotic assisted insertion of transabdominal cerclage (RTAC) pre-pregnancy. She subsequently conceived spontaneously and carried the pregnancy to term and delivered a healthy baby via caesarean section. There have been multiple published studies showing that an MIS approach for abdominal cerclage insertion is safe and viable. Robotic assisted procedures allow for better visualisation and manipulation of tissue especially in patients anticipating a complex procedure. For our patient we feel that a robotic assisted procedure would be more beneficial given her profile and the complexity of her case.
\end{abstract}

Keywords: Obstetrics, Clerclage, Pregnancy

\section{INTRODUCTION}

Cervical incompetence is a multifactorial condition characterised by painless dilatation of the cervix which can be due to congenital or acquired causes. It is commonly associated with repeated cervical trauma in the form of cervical lacerations during child birth or cervical operations such as loop electrical excision procedures or those require forced cervical dilatation such as evacuation of uterus. ${ }^{1}$ It is believed to affect up to $1 \%$ of the general obstetric population and is estimated to be responsible for $8 \%$ of women with recurrent second trimester losses. ${ }^{2}$
Cervical cerclage has long been a standard for managing preterm labor and cervical incompetence. It was first proposed by Shirodkar in 1955 and the methods and efficacy of this intervention has been studied and discussed extensively since.

A recent Cochrane review published in 2017 by Alfirevic et al reviewed 15 trials involving over three thousand women with cervical cerclage. The study concluded that pregnant women with cerclage were less likely to have preterm labour compared to controls before 37, 34 and 28 completed weeks of gestation. Cervical cerclage does reduce the risk of preterm birth in woman at high risk of preterm and probably reduces the risk of perinatal deaths as well. ${ }^{3}$ 
A review article published recently in the Obstetrician and Gynaecologist (TOG) in 2016 by Gibb et al lists a grossly disrupted cervix, an absent vaginal cervix and previously failed elective vaginal cerclage as main indicators for a transabdominal cerclage. ${ }^{4}$

In our case above, Mrs. $\mathrm{L} \mathrm{K}$ had repeated second trimester miscarriages despite application of both a Macdonald cerclage and a Shriodkha cerclage respectively. A transabdominal cerclage can be considered in these patients.

The traditional open transabdominal cerclage was first described in 1965 by Benson and Durfee and published case reports over the years cite high neonatal survival rate of between $88 \%$ to $100 \%$ post transabdominal cerclage insertion. $^{4}$

Advancements in minimally invasive surgery allowed for the transabdominal cerclage to be placed laparoscopically for the first time in 1998 and with further advances, the first robotic assisted transabdominal cerclage insertion (RTAC) was performed in 2018.

Patient body habitus restricting instrument mobility and aberrant anatomy obstructing field of view could potentially complicate an MIS approach to surgery.

In view of the complexity of our case along with our patient profile we decided to offer our patient Mrs. L K a RTAC, utilising technology to help achieve a good outcome for our patient.

\section{CASE REPORT}

We present a case of Mrs. L. K. who underwent RTAC in 2018. Mrs. L K is a thirty-one-year-old gravida 3 para 0 married lady who has a history of three consecutive mid trimester miscarriages with the latter two having had cervical cerclage inserted antenatally. She also had a laparoscopic ovarian cystectomy in 2008 for right ovarian dermoid cyst with no other significant past medical history.

Her first miscarriage occurred at 23 weeks of gestation when she was in her early twenties. She presented to a private hospital with painless cervical dilatation and subsequently miscarried.

In view of her previous second trimester miscarriage, she was placed under cervical length surveillance and managed in the high risk obstetric clinic for her second pregnancy a year later. Cervical dilatation was noted as early as at her 16-week scan. An emergency cerclage (MacDonald cerclage) was subsequently inserted at 17 weeks of gestation when she presented with vaginal bleeding and increased vaginal discharge. Mrs. L. K. was monitored inpatient post procedure but developed chorioamnionitis three weeks post cerclage insertion. The cerclage was cut and Mrs. L. K. miscarried on her twentieth week of gestation.

A year later, Mrs. L. K. conceived spontaneously and an early elective Shriodkar cerclage was inserted at 13 weeks of gestation. She subsequently presented 9 weeks later with increasing contraction pain. She miscarried two weeks later at 23 weeks of gestation and had a surgical evacuation of uterus for adherent placenta.

In preparation for her fourth pregnancy, a plan was made to insert an abdominal cerclage pre - pregnancy so as to reduce her risk of miscarriage.

Baseline ultrasound scans were unremarkable apart from multiple fibroids with the largest being a $3 \mathrm{~cm}$ anterior subserosal fibroid. Mrs. L. K. was then thirty years old with a body mass index (BMI) of 35.3. (height $154 \mathrm{~cm}$, weight $83.8 \mathrm{~kg}$ ). Robotic insertion of abdominal cerclage was offered in view of her body habitus, high BMI and also presence of multiple uterine fibroids. Mrs. L. K. was counselled on the risks and benefits of robotic surgery including the risk of conversion to laparotomy. She was also informed that a successfully inserted abdominal cerclage will not completely negate the possibility of another second trimester miscarriage.

Mrs. L. K. was asymptomatic from her subserosal fibroids and was not recommend a myomectomy. After careful consideration and discussion, Mrs. L. K. decided to proceed with the procedure.

\section{Operative details}

Mrs. L. K. underwent a robotic assisted insertion of transabdominal cerclage (RTAC) at Singapore General Hosptial. She was placed in lithotomy position and catheterised pre operatively. A dose of prophylactic intravenous cefazolin was administered prior to port insertion. An Advincula arch with tip size $8 \mathrm{~cm}$ was used to manipulate the uterus.

She had a total of four ports inserted - one camera port through the umbilicus, the first working port at the right iliac fossa followed by the second working port at the left iliac fossa. A $12 \mathrm{~mm}$ assistant port was inserted last at the right side level of the umbilicus in between the two working ports.

Intraoperatively, she was found to have a $5 \mathrm{~cm}$ subserosal anterior fibroid and $5 \mathrm{~cm}$ intramural posterior fibroid. Bilateral fallopian tubes and ovaries were found to be normal.

A monopolar scissors was used in the first robotic arm and a Maryland bipolar was attached to the second robotic arm. Procedure was commenced with the separation of the uterovesical fold followed by the identification and skeletanisation of the uterine arteries at 
the level of the isthmus at the junction of the ascending and descending branches of the uterine artery.

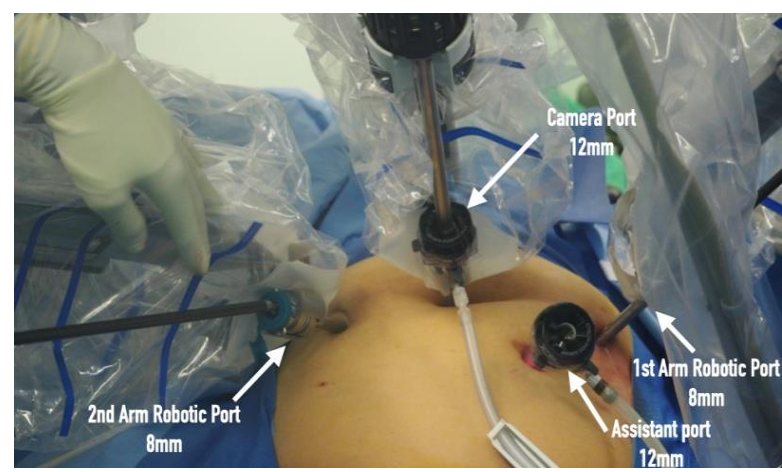

Figure 1: Overview of the robotic ports used.

Mersilene tape was then introduced via the assistant port. The needle was threaded through between the isthmus and the uterine artery to avoid incorporating the myometrium of the uterus into the cerclage and also to reduce bleeding.

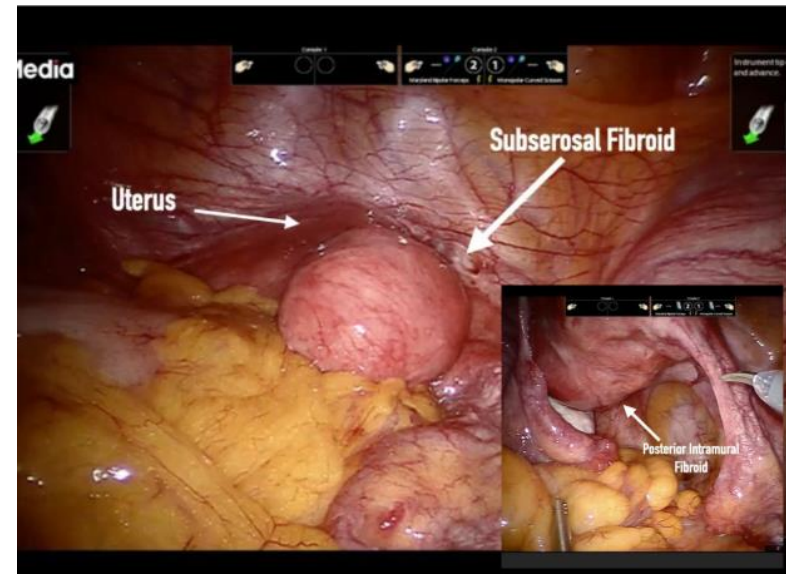

Figure 2: A $5 \mathrm{~cm}$ subserosal anterior wall fibroid along with a $\mathbf{5} \mathbf{~ c m}$ intramural posterior wall fibroid obstructing access to the uterine isthmus.

The abdominal cerclage was placed at the level of her isthmus. The uterine manipulator was removed prior to tightening of the cerclage knot. A size 3 heigar dilator was inserted as the knot was tightened as a measure to prevent excessive narrowing of the cervical canal. The cerclage was secured via 6 knots placed anteriorly.

The uterovescial fold was sutured back in place over the cerclage knot to bury the knots and to reduce adhesion formation. It could be argued that the surgery could have been made easier with the removal of the obstructing anterior uterine fibroid however this was not recommended to the patient as she was asymptomatic from the fibroid. A myomectomy carried additional risk of bleeding, adhesion formation and also complications associated with morcellation, it also had implications for future pregnancy. Estimated blood loss was 50ml. Duration of operation was.

The ports were removed under direct vision and the assistant port was closed with vicryl 1.0 with endoclose. The remaining ports were closed with monocryl.

Mrs. L K was transferred to the general ward after her operation and had an otherwise uneventful recovery. She was discharged well the next day.

\section{Pregnancy post cerclage insertion}

Mrs. L K was reviewed one month later in the outpatient clinic. Ultrasound scan done confirmed the position of the abdominal cerclage and a cervical length of $3 \mathrm{~cm}$. She reported regular normal menses.

Mrs. L K had a spontaneous conception one year after her abdominal cerclage insertion and was seen at 5 weeks gestational age. She was started on aspirin and oral labetalol for her pre- existing hypertension. Antenatal scans were unremarkable and showed no foetal anomaly. Mrs. L K was also diagnosed with gestational diabetes mellitus but was managed solely with diet control measures.
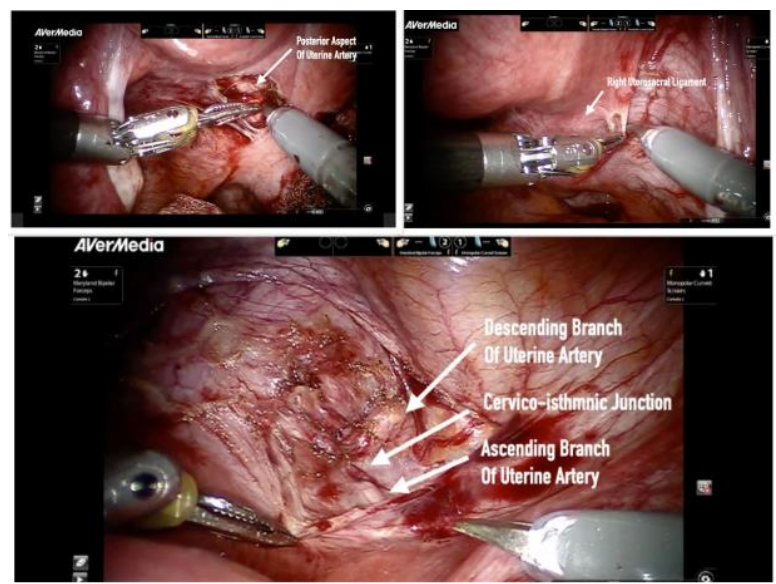

Figure 3: Identification and skeletanisation of the uterine arteries; exposing posterior aspect of uterine artery (top left), identifying right uterosacral ligament

(top right), exposing the branches of the uterine arteries at level of uterine isthmus and correct identification of the cervico- isthmnic junction (bottom).

She had a final growth scan done at 31 weeks with a cervical length of $3.8 \mathrm{~cm}$. Her subserosal uterine fibroids at this point have grown to about $10 \mathrm{~cm}$. Mrs. L K requested for an elective lower segment caesarean section (EL LSCS) due to anxiety from her previous miscarriages and was planned for a EL LSCS at 37 weeks of gestation with intramuscular steriod cover as per Royal College of Obsterician and Gynaecologist (RCOG) guidelines. 
Mrs. L K underwent an uneventful lower segment caesarean section in 2019. A lower segment uterine incision was made above the cerclage and a healthy $3 \mathrm{~kg}$ male baby was delivered with Apgar score 8 and 9 at zero and five minutes respectively. The fibroids previously seen on the scan has grown to a $10 \mathrm{~cm}$ cornual subsersoal fibroid, a $4 \mathrm{~cm}$ right anterior pedunculated fibroid and a 2 $\mathrm{cm}$ intramural fibroid. Bilateral fallopian tubes and ovaries were otherwise normal. The abdominal cerclage was left insitu.

\section{DISCUSSION}

Advancements in minimally invasive surgery have allowed for the transabdominal cerclage to be placed via MIS techniques since 1998 with high neonatal survival rates between $83 \%$ to $95 \%$ post cerclage being reported. ${ }^{6}$

MIS operative techniques are also associated with shorter hospitalisation stay and shorter length of recovery without a large laparotomy scar. ${ }^{4}$ In a study involving 64 women who underwent MIS transabdominal cerclage insertion in Australia, Ades et al reports a mean gestational age of 35.8 weeks with only 1 in 64 women having an adverse intraoperative event, with no postoperative sequelae. ${ }^{6}$

Robotic assisted technology further capitalises on its minimally invasive nature allowing for three dimensional visualisation of anatomy and the use of endowristed instruments. ${ }^{7}$ This improves the ergonomy of surgery and can be a benefit in complex cases such as ours with the obstructing anterior wall fibroid.

A multi centre cohort analysis by Tyan et al published in 2018 studied 68 women who underwent RTAC to assess its feasibility and obstetric benefits in high-risk woman who were projected to have poor pregnancy outcomes. The study reported odds of delivering after 34 and 37 weeks of gestation to be 4- and 3.6-times greater post RTAC. This concluded that RTAC positively influences gestational age and neonatal survival in high-risk obstetric patients. ${ }^{8}$ This study clearly proved that RTAC can positively influence high risk pregnancies.

Obesity is an important determinant of surgical safety and method of surgery. Barbara et al compared pre and postsurgical outcomes of 114 women who underwent a simple hysterectomy in which 50 woman underwent the procedure with robot assistance with the rest undergoing the procedure via laparotomy. The study showed that robotic surgery is associated with shorter inpatient time, reduced blood loss and generally less complications compared to laparotomy. While robotic surgeries are also noted to have longer operative time compared to laparotomy, the difference in operative time becomes non-significant in the subgroup of patients with BMI greater than 35 . This clearly demonstrates the benefits of robotic surgery in cases complicated by high BMI. ${ }^{9}$ For patients such as Mrs. L K with high BMI, robotic surgery can function as a means to undertake complex surgery while preserving an MIS approach reducing intra and post-operative complications.

Our case above demonstrates a positive experience with RTAC. The Robotic approach allowed clear visualisation of pelvic allowing extensive skeletanisation of the uterine vessels at the isthmus and correct placement of the abdominal cerclage. it allowed us to avoid incorporating too much myometrium into the cerclage bite and may help reduce erosion rate and bleeding.

While obesity and complicated anatomy can restrict patient access to MIS surgeries, this case demonstrates how robotic assisted surgery can act as a bridge to include more patients for MIS surgeries.

\section{CONCLUSION}

The placement of an abdominal cerclage allows for the cerclage to be placed more proximally to the internal os allowing for a more security. However, the greatest advantage demonstrated in our case is that the robotic approach allows us to avoid the risk associated with a laparotomy. RTAC also allows improved manoeuvrability of instruments, surgical ergonomics and three-dimensional visualisation of pelvic anatomy. This minimally invasive approach carried reduced risk to the patient and can be considered in more patients. Robotic assisted transabdominal cerclage insertion (RTAC) is a safe and efficacious procedure that should be considered in patients with repeated miscarriages.

\section{Funding: No funding sources Conflict of interest: None declared Ethical approval: Not required}

\section{REFERENCES}

1. ACOG Practice Bulletin No.142: Cerclage for the management of cervical insufficiency. American College of Obstetricians and Gynecologists. Obstet Gynecol. 2014;123(2 Pt 1):372.

2. Wang HL, Yang Z, Shen Y, Wang QL. Clinical outcome of therapeutic cervical cerclage in short cervix syndrome. Zhonghua fu Chan ke za zhi. 2018;53(1):43-6.

3. Brown R, Gagnon R, Delisle MF, Bujold E, Basso $\mathrm{M}$, Bos $\mathrm{H}$, et al. Cervical insufficiency and cervical cerclage. J Obstet Gynaecol Canada. 2013;35(12):1115-27.

4. Zarko A, Stampalijia T, Medley N. Cervical stitch ( cerclage) for preventing preterm birth in singleton pregnancy. Cochr Syste Rev. 2017.

5. Gibb, D, Saridogan, E. The role of transabdominal cervical cerclage techniques in maternity care. Obstet Gynaecol. 2016;18:117-25.

6. Benson RC, Durfee RB. Transabdominalcervicouterine cerclage during pregnancy for the treatment 
of cervical incompetency. Obstet Gynecol 1965;25:142-55.

7. Ades A, May J, Cade TJ, Umstad MP. Laparoscopic transabdominal cervical cerclage: a 6-year experience. Austra New Zeala J Obstet Gynaecol. 2014;54(2):117-20.

8. Zeybek B, Hill A, Menderes G, Borahay MA, Azodi M, Kilic GS. Robot-assisted abdominal cerclage during pregnancy. J Soc Laparoendosco Surge. 2016;20(4).
9. Tyan P, Mourad J, Wright B. Robot assisted transabdominal Cerlcage for the prevention of preterm birth: A multicenter experience. European J Obstetr Gynecol. 2019;232:70-4.

Cite this article as: Tan YH, Durai S, Devendra K, Ravichandran N. A successful full term delivery in a case of robotic assisted insertion of transabdominal cerclage in an obese woman with multiple uterine fibroids. Int J Reprod Contracept Obstet Gynecol 2021;10:1157-61. 